\title{
ON THE ELIMINATION OF NONLINEAR PHENOMENA IN DC/DC CONVERTERS USING TYPE-2 FUZZY LOGIC CONTROLLER
}

\author{
Djamal GOZIM $^{1}$, Kamel GUESMI ${ }^{2}$, Djilali MAHI ${ }^{3}$ \\ ${ }^{1,2,}$ Department of Electrical \& Engineering, University of Ziane Achor Djelfa, Algeria, \\ gozimansour@gmail.com CReSTIC-Reims University, France, kamel.guesmi@univ-reims.fr \\ ${ }^{3}$ Department of Electrotechnical, University Amar Telidji, Laghouat, Algeria, mahidjillali@yahoo.fr
}

\begin{abstract}
DC/DC converters are wealthy of nonlinear phenomena that appear when the converter parameters are subject to perturbation or variation. The converter may exhibit bifurcation from behavior to another, quasi periodic and chaotic responses. In such cases, it is difficult and even impossible to analyze, to predict and to control the converter behavior. This paper gives a description of a DC/DC converter and shows their desirable and undesirable behaviors; then a solution, based on type-2 fuzzy logic controller, is proposed to eliminate the undesirable behaviors and to enhance the converter dynamics.
\end{abstract}

Keywords: Power converter, Type-2 fuzzy logic controller, Bifurcation, Chaos.

\section{INTRODUCTION}

The power converters are used in many fields to adapt the electrical power to the consumer need with minimum loss of energy. The DC/DC converters ensure this task in many fields such as renewable energies, electronic circuits, medical equipment, satellite...etc. However, in some circumstances the variation of the circuit parameters, the load or the power supply perturbations can lead to the apparition of some nonlinear phenomena such as double periodicity, bifurcation and chaos that can destroy the control performances.

Hence, many approaches have been developed, in the literature, to shift or to suppress these nonlinear phenomena [1-4]. Nevertheless, most of these approaches focus on abnormal behaviors removal in a given operating point without any guarantee about the control performances.

The type- 1 fuzzy logic theory has been used in many studies to illuminate the nonlinear phenomena in DC-DC converters [5-10].

In [5], a fuzzy PID controller is synthesized, by analogy with conventional PID, for the regulation of DC/DC converters output voltage. The idea behind is to determine the fuzzy controller parameters based on an established analogy with a conventional PID. This study is extended in [6] to be a systematic approach for synthesizing fuzzy PID controller. It gives possibility of finding or locating different zones of stability of the closed loop system. Other studies followed are focused on finding an optimal choice for the fuzzy controller parameters in the stability zones [7], [8].

The study in [7] proposes a new fuzzy logic controller optimized by the LMI (Linear Matrix Inequality) approach. However, this method is complex and needs an important computation time and memory space for data processing and storage.

In [9], the authors proposed a new approach based on the analytical and systematic calculation of various fuzzy controller parameters to ensure the stability of the converter. The developed controller allows the shifting of the nonlinear phenomena and forces the converter to operate in the simplest behavior in a wide range of variation of the operating point.

To the same end, the authors in [10] proposed the enhancement of the converter behavior by taking into account the effect of perturbation and parameters variation. They used instead of constant reference, a dynamical ramp to dump the effect of perturbation and to keep the simplest behavior of the converter despite of the system parameters variation.

However, the aforementioned works are based on analytical solutions and they are a little bit complex and their implementation in real plant is questionable. In addition, they do not take into consideration the different uncertainties in the converter model and in the definition of the control strategy.

Furthermore, the use of type-1 fuzzy logic in the aim of controlling dynamical electrical systems needs an accurate knowledge on the system to determine the membership functions and to express the control strategy in an optimal number of fuzzy rules.

Furthermore, the membership grade, in type-1 fuzzy system, is a crisp number and cannot handle the different uncertainties. Indeed, uncertainties are of multiples sources and words in fuzzy rules could mean various things to several peoples. To tackle this problem, the type-2 fuzzy logic is proposed as an extension to type-1 fuzzy system. It is 
characterized by a set of membership functions instead of one to describe each situation.

In this study, the Boost converter is selected to be current controlled and functioning in continues conduction mode. This choice is motivated by the fact that the converter, under these conditions, exhibits a large spectrum of nonlinear phenomena. At first, the description of this converter is presented. Then, the proposed method based on type-2 fuzzy logic is described to the end of nonlinear phenomena suppressing. The approach will be validated through simulation results and their performances will be evaluated throughout a comparative study.

\section{BOOST CONVERTER}

The simplified version of the current controlled Boost converter is given in figure 1. In this control mode, we are able to control both slow and fast dynamics of the system [12, 13]. The converter elements are chosen in such manner that the inductor current never drops to zero $\frac{2 L}{R T}>\frac{4}{27}$, and to ensure that the converter operate in continuous conduction mode (CCM) [12]. In this case, we have only two configurations related to the switch $s w$ position.

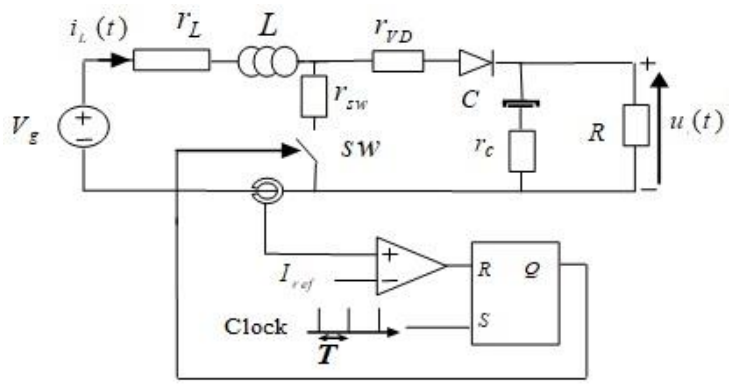

Fig. 1. Boost converter under current mode control (simplified version)

If $T$ is the clock cycle, the dwell times in the two configurations are respectively $t_{1}=d T$ and $t_{2}=d^{\prime} T=(1-d) T$ and the duty cycle $d=t_{1} / T$ is given by this expression:

$$
d(n)=\frac{L}{T\left(r_{L}+r_{s w}\right)} \ln \left(\frac{V_{i n}-\left(r_{L}+r_{s w}\right)-i_{L}(n)}{V_{i n}-\left(r_{L}+r_{s w}\right) I_{r e f}}\right)
$$

The system state, in each configuration, is expressed as:

$$
\dot{x}_{i}=A_{i} x_{i}+B_{i} U
$$

with $A_{i}$ the state matrices in the $i^{\text {th }}$ configuration given by:

$$
\begin{gathered}
A_{1}=\left[\begin{array}{cc}
-\frac{1}{C(R+r c)} & 0 \\
0 & -\frac{r_{L}+r_{S W}}{L}
\end{array}\right], \\
A_{2}=\left[\begin{array}{cc}
-\frac{1}{C\left(R+r_{C}\right)} & \frac{R}{C\left(R+r_{C}\right)} \\
-\frac{R}{L\left(R+r_{C}\right)} & -\frac{r_{L}+r_{V D}+\frac{R r_{C}}{R+r_{C}}}{L}
\end{array}\right], \\
B_{1,2}=\left[\begin{array}{ll}
0 & \left.\frac{1}{L}\right]^{t}
\end{array}\right.
\end{gathered}
$$

and the state vector is $x=\left[v_{c} i_{L}\right]^{t}\left(v_{c}:\right.$ voltage across capacitor, $i_{L}$ : inductor current)

Using the control scheme of figure 1 , it is well known that the converter is wealthy of nonlinear phenomena and exhibits complex and undesirable behaviors [11, 12]. Our goal is to suppress these nonlinear phenomena and to keep the converter operating in the simplest behavior without neglecting the traditional goals of the regulation problem.

The type-1 fuzzy logic controller has been used successfully to this end in several works of our team $[5,6,7,8]$; however, this kind of controller cannot handle efficiently the uncertainties of both system and control strategy. The uncertainty of this last is an inherent characteristic especially in the fuzzification part (choice of the membership functions and their distribution) and in the inference part due to its linguistic nature (the same world have different meanings for different peoples). To solve this problem, we propose in this work to take advantage of the ability of type 2 fuzzy sets theory to include the different uncertainties in the control strategy.

\section{TYPE-2 FUZZY LOGIC CONTROLLER}

The architecture of type-2 fuzzy system is similar to type- 1 with an additional bloc called type reducer. It allows reduction of conclusions from type 2 to type- 1 .

For each linguistic value, the type-2 fuzzy system is characterized by a set of membership functions, instead of one used in classical type-1 fuzzy systems. Hence, the membership grade for every element is a fuzzy set in $[0,1]$. That is unlike the type-1 fuzzy system, where the membership grade is a crisp number [16]. Thus, a type-2 fuzzy system is very useful in circumstances where we need to take into consideration the different uncertainties on converter parameters and on control strategy.

The core of a Type- 2 fuzzy controller is the type-2 fuzzy system given in figure $2[14,16]$. It is constituted of four blocks; the three blocks of type1 fuzzy and a fourth block that ensures the reduction from type-2 fuzzy system to a type-1. 


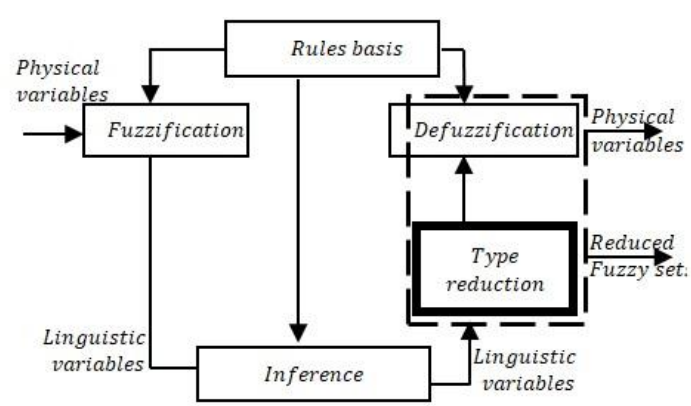

Fig. 2. Structure of Type-2 fuzzy system

\section{TYPE-2 FUZZY CONTROL OF DC-DC CONVERTER}

The significant parameters in current mode control of the Boost converter are the error $e$ between the inductor current and the reference and its time derivative $d e$. These parameters are the input of the fuzzy controller. The output of this last is the increment of the control action $d c_{n}$. The type-2 fuzzy controller scheme is shown in figure 3.

With

$e(k)=I_{\text {ref }}(k)-i_{l}(k) ; d e(k)=e(k)-e(k-1)$

$d c:$ Increment of control action

$K_{e}, K_{d e}, K_{d c}:$ Normalization gains

\subsection{Fuzzification}

For fuzzification, we use the triangular and trapezoidal membership functions for inputs and singleton for output (see figure 4).

The linguistic variables used for fuzzification are: NB (Negative Big); NS (Negative Small); Z (Zero); PS (Positive Small); PB (Positive Big).

\subsection{Inference}

The inputs variables of the type-2 fuzzy controller have five fuzzy sets, which give twentyfive rules for the highest membership functions, and twenty-five rules for the lower limit of the membership functions. The flowing table summarizes the different rules of the control strategy obtained by the interconnection between the input variables.

The membership functions are determined by the following expression:

$$
\mu_{R E S}\left(x_{r}\right)=\frac{1}{m} \sum_{i=1}^{m} \mu_{c i} C_{i}
$$

Where $m$ the number of actives rules and $C_{i}$ the output fuzzy sets (singletons).

$$
\mu_{c i}=\left\{\begin{array}{l}
\mu_{u i}\left(x_{1}\right) \cdot \mu_{u i}\left(x_{2}\right) \\
\mu_{l i}\left(x_{1}\right) \cdot \mu_{l i}\left(x_{2}\right)
\end{array}\right.
$$

$\mu_{u i}$ and $\mu_{l i}$ are, respectively, the upper and lower membership functions.

The inference mechanism of type-2 fuzzy logic is explained in figure 5 .

\subsection{Defuzification}

The final output of the type-2 fuzzy controller $\left(\boldsymbol{d} \boldsymbol{c}_{\boldsymbol{n}}\right)$ is equal to the average of the both decisions for the high and low limits of membership function given by this expression:

$$
d c_{n}=\frac{\left(d c_{h n}+d c_{b n}\right)}{2}
$$

with

$$
d c_{h n}=\frac{\sum_{i=1}^{m} \mu_{h c i} C_{h i}}{\sum_{i=1}^{m} \mu_{h c i}} ; d c_{b n}=\frac{\sum_{i=1}^{m} \mu_{b c i} C_{b i}}{\sum_{i=1}^{m} \mu_{b c i}}
$$

\section{SIMULATION RESULTS}

To obtain the global picture on the different behaviors of the Boost converter and to validate the proposed type- 2 fuzzy controller, we used the bifurcation diagram tool. In this context, we are interested in building the bifurcations diagram in the case of the input voltage variation, load and reference current variation. We evaluate, after that, the proposed approach performance through a comparative study with the obtained results in previous works.

The Boost converter parameters are: $V_{e}=30 \mathrm{~V}, L=27 \mathrm{mH}, R=20 \Omega, C=120 \mu \mathrm{F}$, $r_{l}=1.2 \Omega, r_{c}=0.1 \Omega, r_{s w}=0.3 \Omega, r_{v d}=0.24 \Omega$ and $f_{s w}=1 / T=500 H z$.

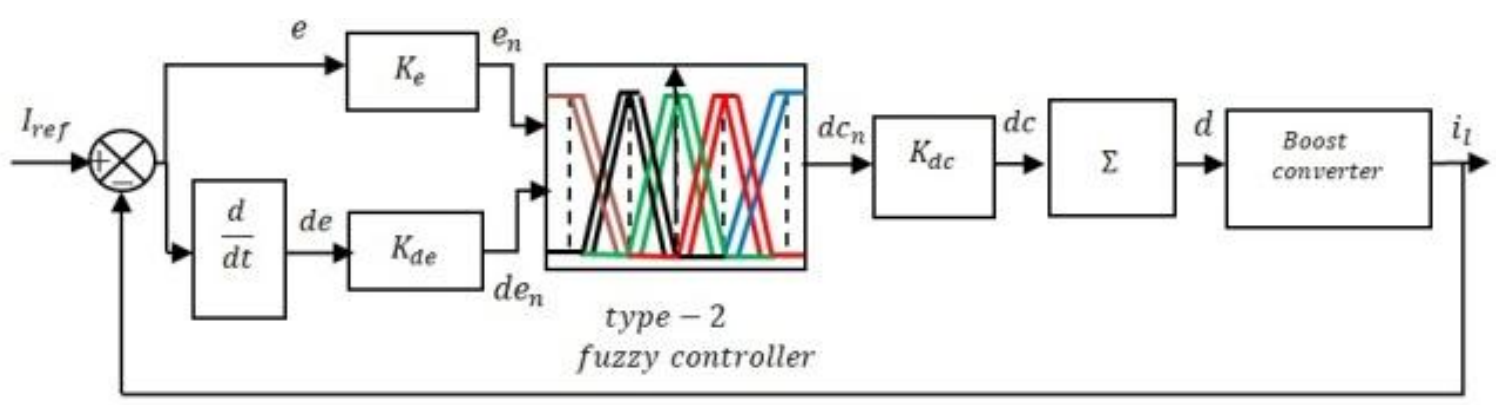

Fig. 3. Scheme of Type-2 fuzzy controller of the Boost converter 


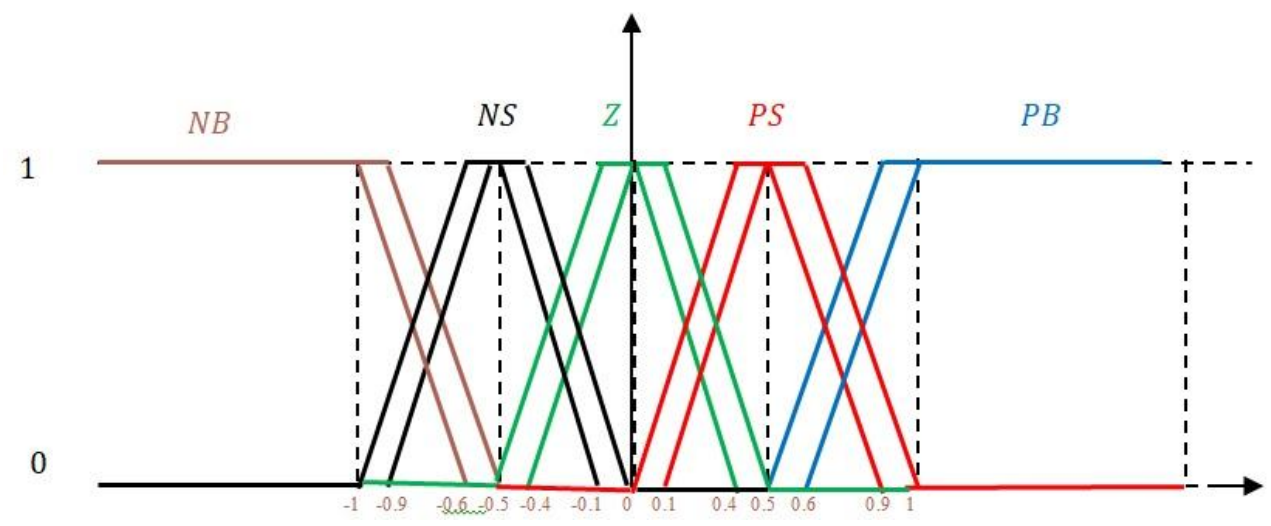

(a) Membership functions for inputs

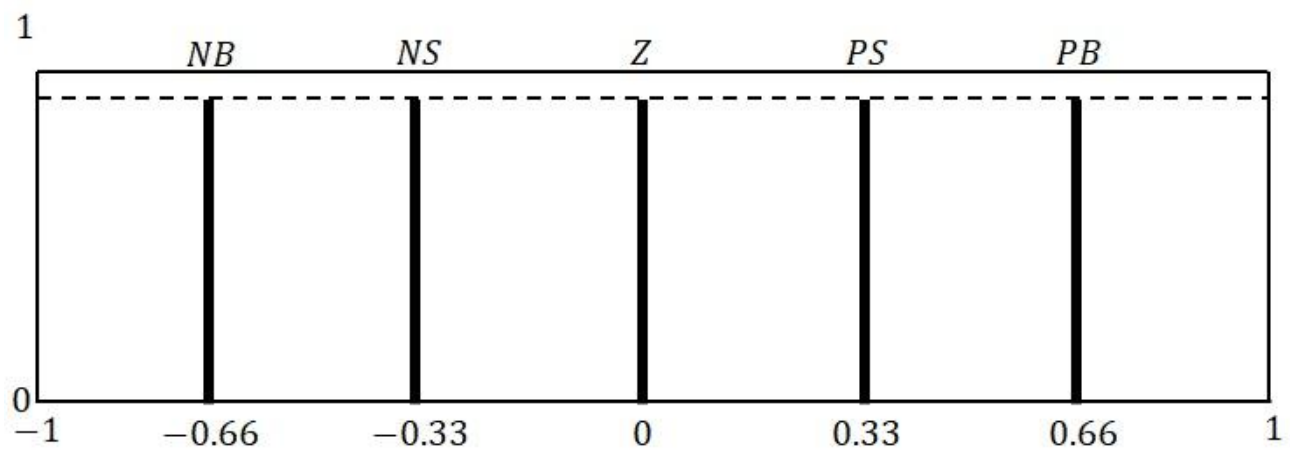

(B) Singletons of the output

Fig. 4. Inputs and output membership functions

Table 1. Inference matrix

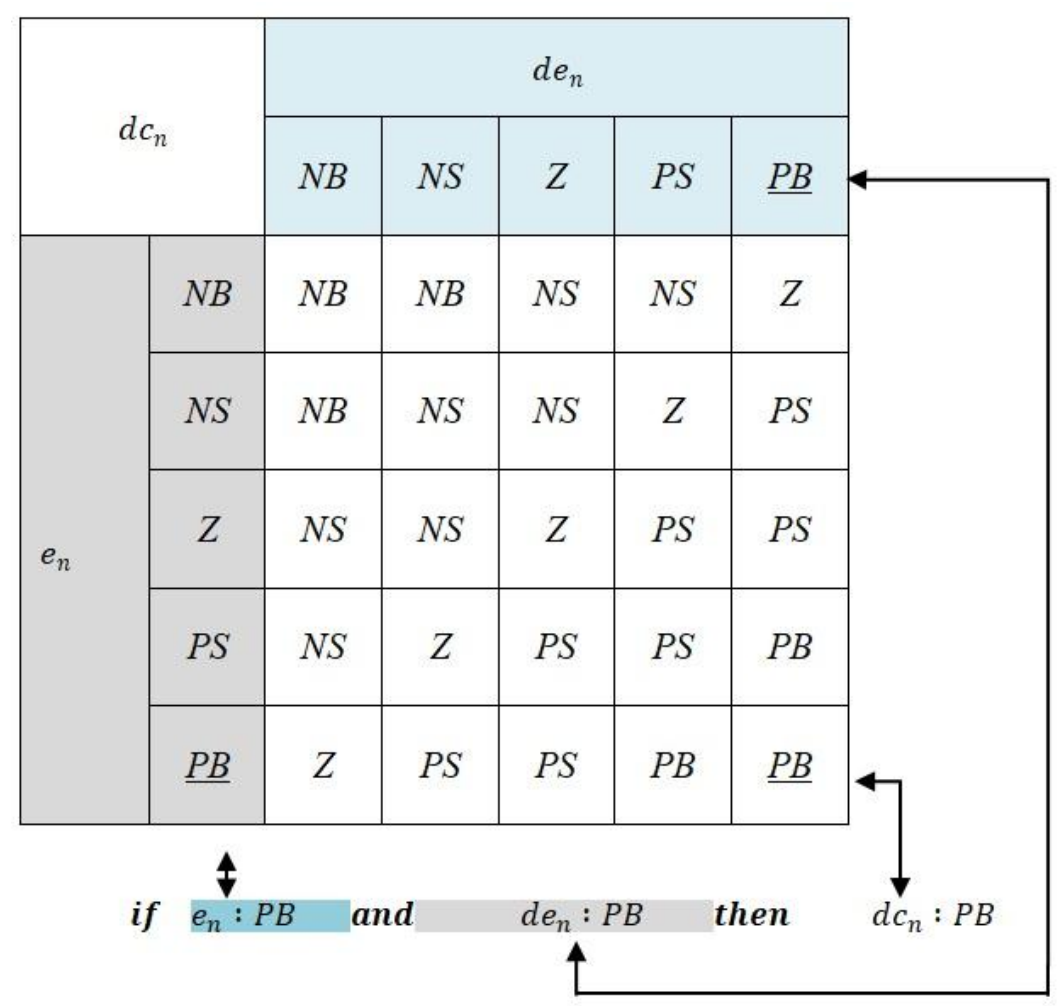




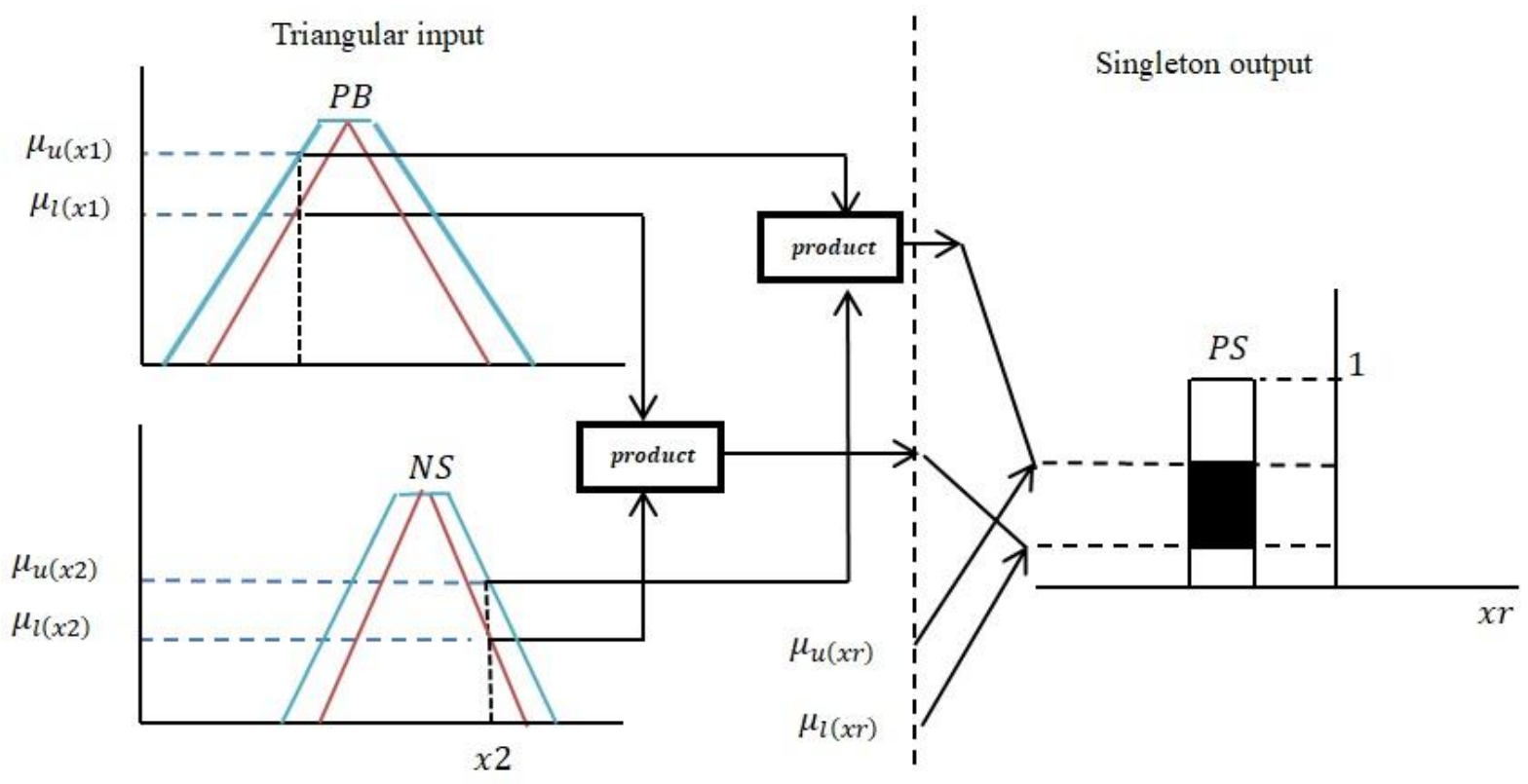

Fig. 5. Inference mechanism

\subsection{Bifurcation diagram with reference current variation}

The figure 6 shows the converter behavior in the case of reference current variation.

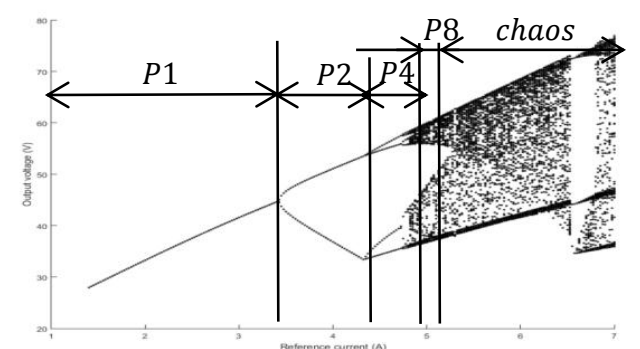

a) Original behavior

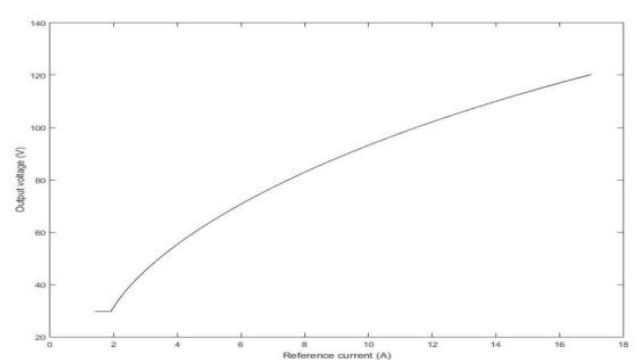

b) Behavior with type-2 fuzzy controller

Fig. 6. Bifurcation diagrams with reference current variation

The Figure 6a illustrates the original behavior of the Boost converter and gives the different operating zones $\mathrm{P}_{1}, \mathrm{P}_{2}, \mathrm{P}_{4}, \mathrm{P}_{8}$ and chaos. Figure $6 \mathrm{~b}$ shows the enhancement obtained by the proposed Type-2 fuzzy controller. We remark that the proposed controller allows the illumination of the undesirable phenomena and the widening of the desired area of period 1 . Indeed, the simplest behavior "period 1" is enlarged from [1.4-3.5]A (period-1) in the original behavior to [1.4-16]A under the use of the proposed type-2 fuzzy controller.

\subsection{Bifurcation diagram with input voltage variation}

In the case of the input voltage variation, the obtained bifurcation diagrams are represented in figure 7 .

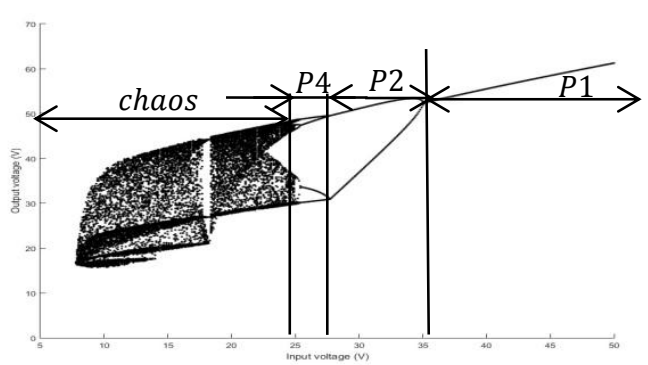

a) Original behavior

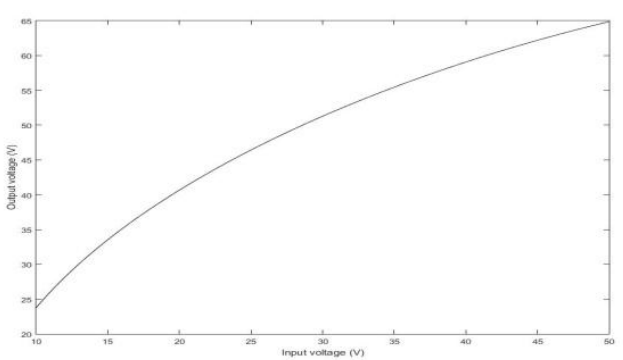

b) Behavior with type-2 fuzzy controller

Fig. 7. Bifurcation diagrams with input voltage variation

Comparing the two bifurcation diagrams of figure 7 , we can remark the enhancement introduced by the type- 2 fuzzy controller. It allows the elimination of the complex undesirable 
behaviors and ensures a wide region of operating in period 1 behavior. Indeed, the desired zone of period one is extended from the range [35-50] $\mathrm{V}$ in the original behavior to the interval [10-50] $\mathrm{V}$ using type-2 fuzzy controller.

\subsection{Bifurcation diagram with load variation}

In the case of load variation, the Boost converter behaviors with and without the proposed controller are depicted in figure 8.

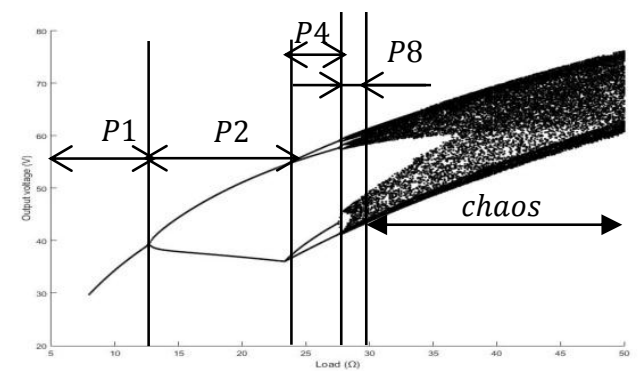

a) Original behavior

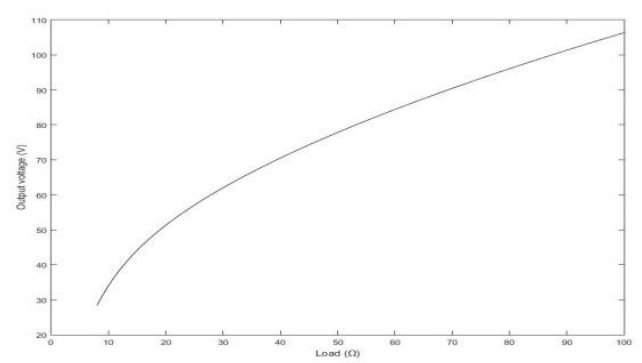

b) Behavior with type-2 fuzzy controller

Fig. 8. Bifurcation diagram with load variation

Figure $8 \mathrm{a}$ shows that the converter exhibit multiple behaviors $\mathrm{P}_{1}, \mathrm{P}_{2}, \ldots$, quasi-periodicity and chaos. The simple and desired behavior is ensured only in a restricted region from $8 \Omega$ to $13 \Omega$; whereas, from figure $8 \mathrm{~b}$, we can remark that the type-2 fuzzy controller ensures the behavior of period 1 (desired behavior) on the whole range and even extend this simple behavior until $\mathrm{R}=100 \Omega$.

\section{EVALUATION OF THE PROPOSED APPROACH}

To evaluate the enhancement introduced by the proposed type- 2 fuzzy controller, we present, in the following, a comparative study with recent results from the literature.

We compared, at first, our results with those obtained by the Type- 1 fuzzy controller presented in [5]. We remark, from figure 9, that the proposed type-2 fuzzy controller ensures a largest zone of period-1 compared to the one obtained by type- 1 fuzzy logic controller, which demonstrate the superiority of the proposed controller in terms of handling the system parameters variation and keeping the system on its simplest behavior.

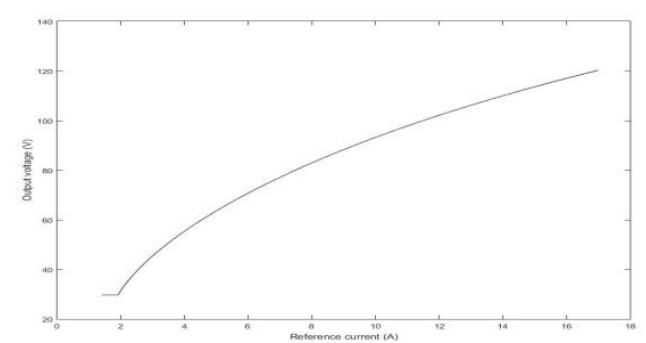

a) Behavior with type-2 fuzzy controller

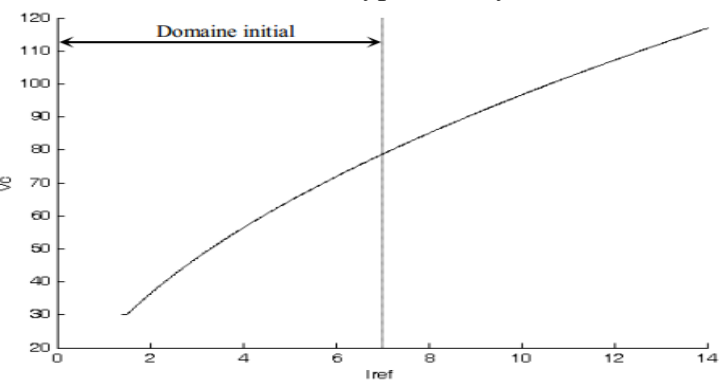

b) Behavior with type-1 fuzzy controller (figure from [5])

Fig. 9. Comparison between type-2 and type-1 fuzzy controllers

Furthermore, if we compare the results obtained by the proposed type-2 fuzzy controller (Figure 10a) with those obtained in [7] using the type-1 fuzzy controller optimized by LMI method (Figure $10 \mathrm{~b}$ ), we remark that not only the performance of the converter are enhanced but also we gain the simplicity of the proposed approach. Indeed, without any complicated optimization task we have a better performance.

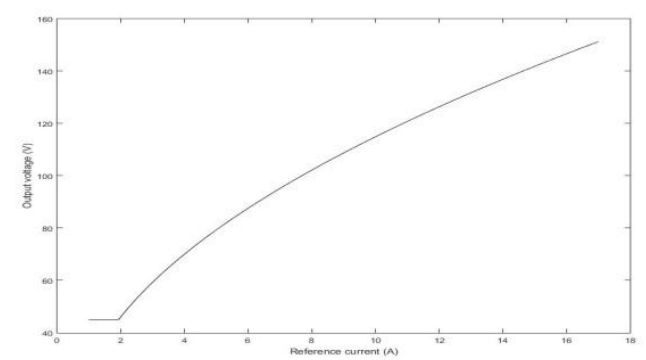

a) Behavior with type-2 fuzzy controller

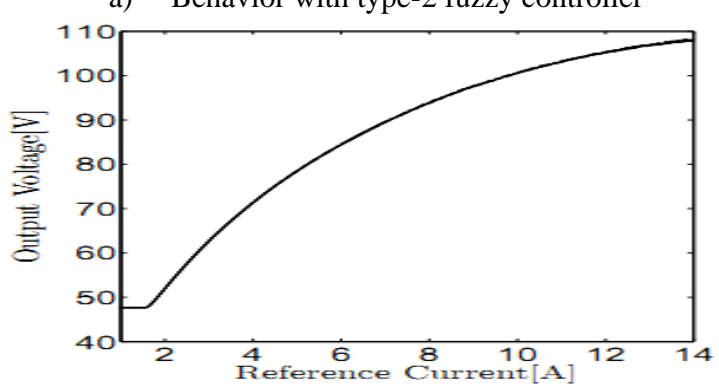

b) Behavior with type-1 fuzzy controller optimized by LMI (figure from [7])

Fig. 10. Comparison between type-2 fuzzy controller and type-1 fuzzy controller optimized by LMI 


\section{CONCLUSION}

In this work, type-2 fuzzy logic controller is proposed for suppressing the nonlinear phenomena exhibited by the Boost converter. This last showed to be wealthy of nonlinear phenomena that complicate the system behaviour and makes their analysis and control an arduous task. Based on type-2 fuzzy logic, the proposed controller allowed handling efficiently the system parameters variation and ensuring the converter operation in its simplest and predictable behavior. The simulation results confirmed this fact and showed the enhancements obtained by the proposed controller in terms of elimination of the undesired complex phenomena. The obtained performances are evaluated through a comparison with those obtained in the literature. These comparisons showed the superiority of the proposed type-2 fuzzy controller against optimized and non-optimized type-1fuzzy logic controllers in term of suppressing nonlinear phenomenon and widening the desired behavior region.

\section{REFERENCES}

1. Chen G, Dong X. From chaos to order: Perspectives, Methodologies, and Applications, Series on Nonlinear Science Series, 1998.

2. Chen G. Controlling chaos and bifurcation in engineering systems. Boca Raton, FL, USA: CRC Press, 1999.

3. Tse CK, Bernardo M. Complex Behavior in Switching Power Converters. IEEE Proceeding, 2002; 5: 768781.

4. Fradkov AL, Evans RJ. Control of chaos: methods and applications in engineering. Annual Review in Control, 2005; 29(1): 33-56.

5. Guesmi K, Essounbouli N, Hamzaoui A, Zaytoon J, Manamanni N.S hifting nonlinear phenomena in a DC- DC converter using a fuzzy logic controller. Journal of mathematics and computers in simulation, 2008; 76(5): 398-409.

6. Guesmi K, Essounbouli N, Hamzaoui A. Systematic design approach of fuzzy PID stabilizer for DC-DC converters. Energy Conversion and Management, 2008; 49(10): 2880-2889.

7. Kamyar M, Damian G, Bashar Z. Stability analysis and control of nonlinear phenomena in boost converters using model-based Takagi-Sugeno fuzzy approach. IEEE Transactions on Circuits and Systems-I: Regular Papers. 2010;57(1): 200-212. https://doi.org/10.1109/TCSI.2009.2019389

8. Khoudiri A, Guesmi K, Hamzaoui A, Essounbouli N, Mahi D. Optimized sliding mode control for DC-DC converters using simplex-PSO techniques. 3rd International Conference on Systems and Control. 2013; 502-507.

9. Guesmi K, HamzaouiA. On the modeling of DC/DC converters: An enhanced approach. International Journal of Numerical Modelling. 2010; 24(1): 36-57. https://doi.org/10.1002/jnm.759

10.Guesmi K, Gozim D, Mahi D, Hamzaoui A. Dynamical ramp for nonlinear phenomena control in DC-DC converters. Journal of Electrical Systems, 2010.

11. Banerjee S, Verghese GC. Nonlinear phenomena in power electronics. Wiley-IEEE Press, USA, 2001.
12. Erickson R, Maksimovic D. Fundamentals of power electronics. Kluwer Academic Publishers, Boston, Dordrecht, London, 1999.

13. Hagras H. Type-2 FLCs: A new generation of fuzzy controllers. IEEE Computational Intelligence Magazine. 2007; 2(1): 30-43.

14. Mendel JM. Type-2 fuzzy sets and systems. IEEE Computational Intelligence Magazine. 2007; 2(1): 20-29.

15. Atacak I, Bay O. A type-2 fuzzy logic controller design for buck and boost DC-DC converters. Journal of intelligent manufacturing, 2012: 1023- 1034. https://doi.org/10.1007/s10845-010-0388-1

16. Mendel JM. Type-2 Fuzzy sets: some questions and answers. IEEE Neural Networks Society. 2003;1:1013.

17. Castillo O, Melin P. Recent advances in interval type2 fuzzy systems. Springer in Computational Intelligence. 2012; 1.

18. Castillo O. Type-2 fuzzy logic in intelligent control applications. Springer, 2012; 272. https://doi.org/10.1007/978-3-642-24663-0_1

19. Greenfield S. Type-2 fuzzy logic: Circumventing the defuzzification bottleneck. Thesis at University of Montfort, 2012.

20. John RI, Coupland S. Type-2 Fuzzy logic a historical view. IEEE Computational Intelligence Magazine. 2007; 2(1): 57-62.

21. Gozim D, Guesmi K, Mahi D. On the enhancement of DC-DC converters behavior. Przegląd elektrotechniczny. 2013; 89(7): 45-49.

Received 2018-04-19

Accepted 2018-07-11

Available online 2018-07-19

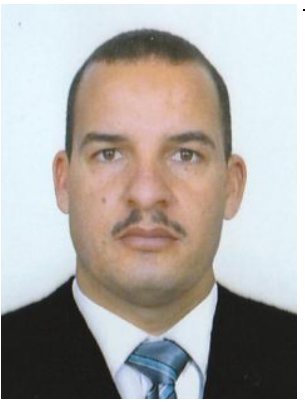

Dr. Djamal GOZIM, Was born in Djelfa-Algeria, in 1980. $\mathrm{He}$ received a Magister from polytecnical militery school in 2006, PhD degrees in Electrical Engineering from University Amar Telidji - Laghouat, Algeria, He works in University of Djelfa, Algeria, Faculty of sciences and technology, Email:gozimansour@gmail.com

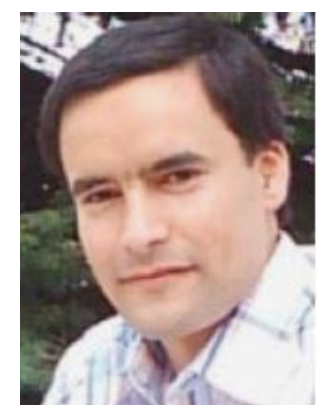

Prof. Kamel GUESMI, received the BEng degree in Electrical Engineering from the university of Djelfa, Algeria, in 2000, the MSc and PhD degrees in Electrical Engineering from Reims University, France, in 2003 and 2006, respectively. Currently, he is a full Professor with the Department of Electrical Engineering, Djelfa

University, Algeria. His research interests include intelligent control, robust control, power electronics and nonlinear dynamics. E-mail:guesmi01@univ-reims.fr 
